Discussion Paper \#2005-2

Inefficiency in a Bilateral Trading Problem with Cooperative Investment

by

Kazumi Hori

May, 2005 


\title{
Inefficiency in a Bilateral Trading Problem with Cooperative Investment*
}

\author{
Kazumi Hori ${ }^{\dagger}$
}

May 2005

\begin{abstract}
A bilateral trading model with investment is considered. In the "cooperative" investment version of the model, the seller's investment stochastically determines the buyer's valuation of the good. The value and cost of the good are realized only after the investment is made, and the investment level and the realization of the good's value and cost are private information. I show that under these assumptions, no contract made prior to the investment can simultaneously induce efficient investment and efficient ex-post trade when the buyer's type is continuously distributed. This inefficiency result contrasts sharply with the efficiency result under the standard "selfish" investment model, where the seller's investment stochastically determines the seller's cost.
\end{abstract}

Keywords: Bilateral Trading, Cooperative Investment, Contracts. JEL Classification Numbers: C72, D23, D82.

${ }^{*}$ This note is based on the third chapter of my Ph.D. dissertation at University of Wisconsin-Madison. I would like to thank Yeon-Koo Che, my adviser, for suggesting this problem, and Larry Samuelson, William Sandholm, Lucía Quesada and Donald Hausch for helpful comments.

${ }^{\dagger}$ Graduate School of Economics, Hitotsubashi University. Address: 2-1 Naka, Kunitachi, Tokyo 186-8601, Japan. Phone: 81-42-580-8505. E-mail: khori@econ.hit-u.ac.jp. Web page: http://www.econ.hit-u.ac.jp/ khori. 


\section{Introduction}

Consider the following interaction between a buyer and a seller. The buyer is interested in obtaining one unit of a good that the seller can produce. The seller makes an investment which stochastically determines the buyer's valuation of the good; in this sense the investment is "cooperative." The good is traded after its value and cost are realized.

The note asks, when the seller's investment level is unobservable and the good's value and cost are private information, whether the first best outcome (defined as simultaneous efficient investment and efficient trade) is possible. This problem is not trivial. If the investment problem is absent, efficient trade is achievable, because, unlike Myerson and Satterthwaite (1983), there is no interim individual rationality. ${ }^{1}$ Also, if the good's value and cost are not private information, efficient investment is attainable.

This note shows that, under a "cooperative" investment environment, no efficiency-inducing contract exists when the buyer's valuation is distributed continuously. Schmitz (2002a) offers a model in which the buyer's valuation of the good is either "high" or "low." He shows that the first-best outcome is possible when the good's cost lies between high and low valuations. The current note finds that this is the only case in which the first-best is possible when investment is "cooperative."

This inefficiency result contrasts sharply with the efficiency result under the standard "selfish" investment model, where the seller's investment stochastically determines the seller's cost. Konakayama et al. (1986) and Rogerson (1992) proved the existence of an efficient mechanism in the standard model; moreover, Schmitz (2002b) showed that the first-best outcome is achievable through a simple contract and renegotiation.

Several papers have analyzed this problem in a complete information framework. Che and Hausch (1999) showed that in a cooperative investment model, the efficient outcome may not be attainable if parties cannot commit not to renegotiate. In contrast, in a selfish investment model under the same assumptions, the first-best outcome may be achievable (Nöldeke and Schmidt 1995, Edlin and Reichelstein 1996). However, very few analysis have employed an incomplete information framework. This note provides insights into the cooperative investment model when the investment level and the realization of the good's value and cost are private information.

\section{Model}

Consider a buyer $(B)$ and a seller $(S)$, both of whom are risk-neutral. In the initial period, $t=0$, they agree to a contract specifying the allocation

\footnotetext{
${ }^{1}$ Myerson-Satterthwaite Theorem shows that it is impossible to achieve ex-post efficiency when the good's value and cost are private information and trade is voluntary.
} 
of one unit of an indivisible good and the associated monetary transfer. At time $t=1$, the seller chooses the investment level. The investment level stochastically determines the realization of the buyer's valuation of the good at time $t=2$ as follows. Suppose the seller chooses an investment level, $e \in E \subset \Re$. The cost of investment is represented by $\psi(e)$, where $\psi_{e}>0$ and $\psi_{e e}>0$. Then the buyer's valuation, $v \in V=[\underline{v}, \bar{v}] \subset \Re$, is realized according to the distribution function $F(v \mid e)$. The distribution function is first-order stochastic dominant, with $F_{e}(v \mid e) \leq 0$ and $F_{e}(v \mid e)<0$ for some $v$. The seller's production cost, $c \in C \subset \Re,{ }^{2}$ is also realized at time $t=2$, according to distribution function $G(c)$. Note that this cost does not depend on the investment level, and thus the investment is "cooperative." It is assumed that $\sup V>\inf C .^{3}$

Let $q(\tilde{v}, \tilde{c})$ and $t(\tilde{v}, \tilde{c})$ be the allocation and transfer rules, respectively, for time $t=3$ as specified by the contract agreed upon in the initial period. $q(\tilde{v}, \tilde{c}) \in[0,1]$ and $q(\tilde{v}, \tilde{c})=1$ when the buyer obtains the good and $q(\tilde{v}, \tilde{c})=$ 0 when the seller keeps the good. A version of the Revelation Principle shows that it is without loss of generality to restrict our attention to direct mechanisms. The allocation and transfer rules are contingent on the reports from the buyer, $\tilde{v} \in V$, and the seller, $\tilde{c} \in C$. The contract can be contingent on the seller's report on investment level, but it is useless because investment does not satisfy single crossing property. Then the utility of the buyer and the seller after time $t=2$ are given by ${ }^{4}$

$$
\begin{aligned}
u^{B}(\tilde{v}, \tilde{c} \mid v) & =v q(\tilde{v}, \tilde{c})-t(\tilde{v}, \tilde{c}) \\
u^{S}(\tilde{v}, \tilde{c} \mid c) & =t(\tilde{v}, \tilde{c})-c q(\tilde{v}, \tilde{c})-\psi(e) .
\end{aligned}
$$

The efficient allocation is then

$$
q^{*}(v, c)=\left\{\begin{array}{l}
1 \text { when } v \geq c \\
0 \text { when } v<c
\end{array}\right.
$$

Intuitively, this condition states that the buyer obtains the good only if her valuation is more that the seller's cost. Note that this condition does not depend on the investment level. Because $\sup V>\inf C$, there exists some valuation-cost pair $(v, c)$ for which $q^{*}(v, c)=1$.

\section{$3 \quad$ The Inefficiency Result}

This section investigates an incentive for investment given an incentive compatible allocation rule.

\footnotetext{
${ }^{2} C$ does not need to be an interval. Also, $c$ does not need to be stochastic.

${ }^{3}$ If $\sup V \leq \inf C$, choosing the lowest investment level is optimal, the first best is attainable.

${ }^{4}$ This paper considers only transfer rules which satisfy a balanced-budget constraint.
} 
Consider an allocation rule, $q=q(v, c)$, which is strictly positive for some $(v, c)$. To achieve this allocation ex-post, the allocation and transfer rules must satisfy the following incentive compatibility constraints at time $t=3$ :

$$
\begin{aligned}
\int_{C}\{v q(v, c)-t(v, c)\} g(c) d c & \geq \int_{C}\left\{v q\left(v^{\prime}, c\right)-t\left(v^{\prime}, c\right)\right\} g(c) d c, \\
\int_{V}\{t(v, c)-c q(v, c)\} f(v \mid e) d v & \geq \int_{V}\left\{t\left(v, c^{\prime},\right)-c q\left(v, c^{\prime}\right)\right\} f(v \mid e) d v, \\
\forall\left\{(v, c),\left(v^{\prime}, c^{\prime}\right)\right\} \in\{V \times C\}^{2} &
\end{aligned}
$$

Given some allocation rule, $q$, let $e^{\dagger}(q)$ be the efficient investment level.

Assumption 1. $e^{\dagger}(q)>\inf E$.

This assumption precludes irrelevant cases.

Assumption 2. The efficient investment level is an inner solution: $e^{\dagger}(q)$ satisfies the first order condition

$$
\int_{C} \int_{V}(v-c) q(v, c) f_{e}\left(v \mid e^{\dagger}\right) g(c) d v d c=\psi_{e}\left(e^{\dagger}\right) .
$$

This assumption is met when $F_{e e} \geq 0$ and $C=\{c\}$, for example.

Proposition 1. Given an allocation rule $q$, any transfer rule $t$ can induce an investment level less than the efficient investment level, $e<e^{\dagger}(q)$, only.

Proof. The proof proceeds by contradiction. Suppose the contract which implements the investment level $e \geq e^{\dagger}(q)$ exists, then the contract should satisfy incentive compatibility conditions and

$$
\int_{C} \int_{V}(v-c) q(v, c) f_{e}(v \mid e) g(c) d v d c \leq \psi_{e}(e) .
$$

The buyer's interim utility at time $t=2$ is

$$
u^{B}(v) \equiv \int_{C}\{v q(v, c)-t(v, c)\} g(c) d c .
$$

From incentive compatibility condition (1), applying the envelop theorem, this interim utility is

$$
u^{B}(v)=u^{B}(\underline{v})+\int_{\underline{v}}^{v} \int_{C} q\left(v^{\prime}, c\right) g(c) d c d v^{\prime},
$$

then the interim transfer is

$$
\int_{C} t(v, c) g(c) d c=\int_{C} v q(v, c) g(c) d c-\int_{\underline{v}}^{v} \int_{C} q\left(v^{\prime}, c\right) g(c) d c d v^{\prime}-u^{B}(\underline{v}) .
$$


Integrating by parts, the expected transfer is

$$
\begin{aligned}
& \int_{V} \int_{C} t(v, c) g(c) f(v \mid e) d c d v \\
& \quad=\int_{V} \int_{C} v q(v, c) g(c) f(v \mid e)-q(v, c) g(c)\{1-F(v \mid e)\} d c d v-u^{B}(\underline{v}) .
\end{aligned}
$$

The seller's expected utility at time $t=1$ is

$$
E u^{S} \equiv \int_{C} \int_{V}\{t(v, c)-c q(v, c)\} f(v \mid e) g(c) d v d c-\psi(e),
$$

and, by substituting (4),

$E u^{S}=\int_{V} \int_{C}(v-c) q(v, c) g(c) f(v \mid e)-q(v, c) g(c)\{1-F(v \mid e)\} d c d v-u^{B}(\underline{v})-\psi(e)$.

The seller's investment maximizes above expected utility, hence the first order condition satisfies

$$
\int_{V} \int_{C}(v-c) q(v, c) g(c) f_{e}(v \mid e)+q(v, c) g(c) F_{e}(v \mid e) d c d v=\psi_{e}(e) .
$$

The second part of the left hand side is strictly negative. Suppose not, $F_{e}(v)=0$ for almost all $\left\{v \mid \int_{C} q(v, c) g(c) d c>0\right\}$, then $f_{e}(v \mid e)=0$ for almost all $\left\{v \mid \int_{C} q(v, c) g(c) d c>0\right\}$, and the lowest investment is efficient, which is the case precluded by the assumption. Hence,

$$
\int_{V} \int_{C}(v-c) q(v, c) g(c) f_{e}(v \mid e) d c d v>\psi_{e}(e)
$$

a contradiction to $(3)$.

Comments are in order. First, uncertainty about $c$ is not essential for the inefficiency result. Second, there is no assumption on ex-post bargaining stage, the result holds for all incentive compatible allocation and transfer rules.

Why the seller under invests? When $v$ is high, the seller has to pay information rent for the buyer. First-order stochastic dominance means that, when the seller invests, probability of realization of high $v$ is large. Because the seller wants to increase the transfer (reduce the payment of information rent), he chooses low investment level.

Next Corollary is immediate from Proposition 1.

Corollary 1. The efficient allocation $q^{*}$ and the efficient investment level $e^{\dagger}\left(q^{*}\right)$ cannot be achieved simultaneously. 


\section{The Second Best Contract}

This section characterizes the second best contract. For simplicity, it is assumed that $C=\{c\}$, there is no uncertainty about the seller's cost.

The following problem, which maximizes the social surplus, is considered.

$$
\max _{q(v), t(v)} \int_{V}(v-c) q(v) f(v \mid e) d v-\psi(e)
$$

subject to

$$
\begin{gathered}
v q(v)-t(v) \geq v q\left(v^{\prime}\right)-t\left(v^{\prime}\right) \quad \forall\left(v, v^{\prime}\right) \in V^{2}, \\
\int_{V}\{t(v)-c q(v)\} f_{e}(v \mid e)=\psi_{e}(e), \\
1 \geq q(v) \geq 0 .
\end{gathered}
$$

The first two constraints are equivalent to monotonicity condition, $q(v) \geq$ $q\left(v^{\prime}\right)$ when $v>v^{\prime}$, and

$$
\int_{V}(v-c) q(v) f_{e}(v \mid e)+q(v) F_{e}(v \mid e) d v=\psi_{e}(e)
$$

as it is shown in the previous section.

Let $\lambda$ be the Lagrange multiplier for (6), then the Hamiltonian is

$$
H \equiv(v-c) q(v)+\lambda\left\{(v-c) q(v)+q(v) \frac{F_{e}(v \mid e)}{f(v \mid e)}\right\},
$$

where $\lambda \geq 0$.

Proposition 2. In the second best contract, $v^{S} \geq c$.

Proof. When $v<c$,

$$
(v-c)+\lambda\left\{(v-c)+\frac{F_{e}(v \mid e)}{f(v \mid e)}\right\},
$$

is always negative, then $q(v)=0$ is the optimal.

$v^{S}<c$ cannot be the second best, not only because it is ex-post inefficient but also reduces the seller's investment by increasing the buyer's information rent. This result is similar to the second best contract of Myerson and Satterthwaite (1983). Although their inefficiency result arises partly because of interim individual rationality, while the current one partly because of cooperative investment, inefficiency is moderate when information rent is modest, which is the result of common problem, incentive compatibility. 
Now, solve the optimal $v^{S}$. Since the Hamiltonian is linear in $q$, the optimal trade decision is characterized by a "bang-bang" solution: ${ }^{5}$

$$
q(v)=\left\{\begin{array}{l}
1 \text { if } v \geq v^{S}, \\
0 \text { if } v<v^{S} .
\end{array}\right.
$$

Given this, (6) is

$$
\int_{v^{S}}^{\bar{v}}(v-c) q(v) f_{e}(v \mid e)+q(v) F_{e}(v \mid e) d v=\psi_{e}(e),
$$

and, integrating by part, this is

$$
\left[(v-c) F_{e}(v \mid e)\right]_{v^{S}}^{\bar{v}}=-\left(v^{S}-c\right) F_{e}\left(v^{S} \mid e\right)=\psi_{e}(e) .
$$

From (7), the investment level can write as a function of $v^{S}, e=e\left(v^{S}\right)$. The problem can now rewrite as

$$
\max _{q(v)} \int_{v^{S}}^{\bar{v}}(v-c) q(v) f\left(v \mid e\left(v^{S}\right)\right) d v-\psi\left(e\left(v^{S}\right)\right) .
$$

Proposition 3. $v^{S}$ is $\bar{v}>v^{S} \geq c$ and a root of

$$
-\left(v^{S}-c\right) f\left(v^{S}\right)+\left\{\int_{v^{S}}^{\bar{v}}(v-c) q(v) f_{e}\left(v \mid e\left(v^{S}\right)\right) d v-\psi_{e}\left(e\left(v^{S}\right)\right)\right\} \frac{\partial e\left(v^{S}\right)}{\partial v^{S}}=0,
$$

where

$$
\frac{\partial e\left(v^{S}\right)}{\partial v^{S}}=-\frac{F_{e}\left(v^{S} \mid e\left(v^{S}\right)\right)+\left(v^{S}-c\right) f_{e}\left(v^{S} \mid e\left(v^{S}\right)\right)}{\left(v^{S}-c\right) F_{e e}\left(v^{S} \mid e\left(v^{S}\right)\right)+\psi_{e e}\left(e\left(v^{S}\right)\right)} .
$$

Proof. The derivative of the objective function is

$$
-\left(v^{S}-c\right) f\left(v^{S}\right)+\left\{\int_{v^{S}}^{\bar{v}}(v-c) q(v) f_{e}\left(v \mid e\left(v^{S}\right)\right) d v-\psi_{e}\left(e\left(v^{S}\right)\right)\right\} \frac{\partial e\left(v^{S}\right)}{\partial v^{S}} .
$$

The derivative is $-(\bar{v}-c) f(\bar{v})<0$, when $v^{S}=\bar{v}$, and

$$
\left\{\int_{c}^{\bar{v}}(v-c) q(v) f_{e}(v \mid e) d v-\psi_{e}(e)\right\} \frac{-F_{e}(c \mid e)}{\psi_{e e}(e)} \geq 0,
$$

when $v^{S}=c$. The bracketed term is positive because Proposition 1 says that the second best investment level is sub optimal. Thus the problem has an solution, $\bar{v}>v^{S} \geq c$, which satisfies the first order condition.

\footnotetext{
${ }^{5}$ See Riley and Zeckhauser (1983).
} 


\section{Conclusion}

It has been shown that it is impossible to achieve the first-best outcome when the buyer's type is continuously distributed. Intuitively, when trade takes place by even or more than two types, same result may apply. This result can be extended to the case where both the buyer and seller make cooperative investments. The first-best outcome is generally impossible in this framework.

\section{References}

Che, Y.-K. and D. B. Hausch (1999), "Cooperative Investment and the Value of Contracting," American Economic Review, 89, 125-147.

Edlin, A. S. and S. Reichelstein (1996), "Holdups, Standard Breach Remedies and Optimal Investment," American Economic Review, 86, 478-501.

Konakayama, A., T. Mitsui and S. Watanabe (1986), "Efficient Contracting with Reliance and a Damage Measure," RAND Journal of Economics, 17, $450-457$.

Myerson, R. B. and M. Satterthwaite (1983), "Efficient Mechanisms for Bilateral Trading," Journal of Economic Theory, 28, 265-281.

Nöldele, G. and K. M. Schimidt (1995), "Option Contracts and Renegotiation: A Solution to the Hold-Up Problem," RAND Journal of Economics, 26, 163-179.

Riley, J. and R. Zeckhauser (1983), "Optimal Selling Strategies: When to Haggle, When to Hold Firm," The Quarterly Journal of Economics, 98, 267-289.

Rogerson, W. P. (1992), "Contractual Solution to the Hold-Up Problem," Review of Economic Studies, 59, 777-793.

Schmitz, P. (2002a), "On the Interplay of Hidden Action and Hidden Information in Simple Bilateral Trading Problems," Journal of Economic Theory, 103, 444-460.

Schmitz, P. (2002b), "Simple Contracts, Renegotiation under Asymmetric Information, and the Hold-Up Problem," European Economic Review, 46, 169-188. 